\title{
A design framework for a home-based stroke rehabilitation system: identifying the key components
}

\author{
Stefan Rennick Egglestone ${ }^{1}$, Lesley Axelrod ${ }^{2}$, \\ Thomas Nind ${ }^{3}$, Ruth Turk ${ }^{4}$, Anna Wilkinson ${ }^{5}$, \\ Jane Burridge ${ }^{4}$, Geraldine Fitzpatrick ${ }^{2}$, \\ Sue Mawson ${ }^{5}$, Zoe Robertson ${ }^{7}$ \\ Correspondence: sre@cs.nott.ac.uk.
}

\begin{abstract}
We present a design framework for a sensor-based stroke rehabilitation system for use at home developed through the analysis of data collected from a series of workshops. Participants had a variety of backgrounds and included people living with stroke and health professionals who work with them. Our focus in these workshops was to learn more about the social context around stroke care, to share early project ideas and develop a design framework for developing systems. In this paper we present a detailed analysis of participant responses and use this analysis to draw specific conclusions about the components and configuration that we believe should be in future systems.
\end{abstract}

Keyword: component; stroke; rehabilitation; framework; home; domestic; pervasive

\section{INTRODUCTION \& BACKGROUND TO WORKSHOPS}

\section{A. Demographics of stroke}

Disabilities caused by stroke include a loss of strength and dexterity and poor balance leading to reduced mobility and risk of social isolation. Stroke is the largest single cause of adult disability in the developed world [32] and in the UK strokerelated services accounted for over $4 \%$ of the NHS budget in 2000 [26]. Stroke is more prevalent in the elderly. Due to the aging population stroke is forecast to create a huge burden of health and social care in the coming decades [37, 43]. As demographic change takes place and the stroke population grows [39] health and rehabilitation services become stretched and delivery of health and social care has to change $[14,15]$. One target is to provide more support for individuals who wish to live independently in their homes [36]. Rehabilitation from stroke can take many years and for some individuals may never be complete so there is a need for home-based rehabilitation.

\section{B. Rehabilitation from stroke}

Stroke is a disease of the central nervous system and impairments are associated with damage to brain tissue. Current research shows recovery is related to neuroplasticity of the brain in which the function of damaged systems is taken on other neural systems. Neuroplastic change is encouraged by progressive physical exercise programmes during which patients is relearn how to use impaired parts of their body [36]. Therapeutic effectiveness depends on intensity and frequency of treatment, especially immediately post-stroke [40].

\author{
Ann Marie Hughes ${ }^{4}$, Kher Hui Ng${ }^{1}$, Will Pearson ${ }^{1}$, \\ Nour Shublaq ${ }^{6}$, Penny Probert-Smith ${ }^{6}$, Ian Rickets ${ }^{3}$, \\ Tom Rodden ${ }^{1}$ \\ Universities of Nottingham ${ }^{1}$, Sussex $^{2}$, Dundee ${ }^{3}$, Southampton ${ }^{4}$, \\ Sheffield Hallam ${ }^{5}$ and Oxford ${ }^{6}$ and Barnsley DGH NHST ${ }^{7}$
}

Clinicians have expertise in encouraging motivation but exercises practiced at home may not be performed as well or as regularly as is needed to facilitate optimum recovery.

\section{Our approach}

Our project, 'Motivating Mobility', seeks to develop technology that will provide support for home-based rehabilitation with a specific focus on stroke and on recovery of the ability to reach and grasp with the shoulder, arm, hand and fingers (referred to throughout the rest of this paper as the "upper limb"). Our broad approach is to develop technology which supports individual motivation to perform exercise, although we are only in the early stages of designing such technology. The workshops described in this paper are part of a research effort to better understand stroke, its social context and how to support motivation in relation to these aspects. We are working within a framework of design which emphasizes collaboration between all stakeholders in the design process and is participatory and user-centered. Our workshops build on earlier work visiting individuals living with stroke in their homes and places where they took part in leisure activities.

\section{Related Work}

There are a number of fields of cross disciplinary related work that we have drawn on. We explored literature from diverse domains within our collaborative multi-disciplinary team and this proved an extremely rich process. Topics included human computer interaction; motivational theories and models; motivation (and barriers to motivation) for therapy, for learning and for interacting with technologies; affective computing; organizational and behavior change and management; psychology of persuasion; choice architecture; serious games, engagement and flow; ludic and persuasive technologies; assistive technologies and telecare; eldercare support and caregiver strain; cognitive behavior therapy and motivational interviewing; advertising; life and sports coaching; virtual rehabilitation, cybertherapy and other technologically assisted therapies and home based systems.

The understandings gained from this broad base contributed to our selection and development of prototypes, protocols, discussion points and topic lists for use at the workshops. We identified a number of key areas of interest that shaped the workshop design. 
We researched clinician's attitudes to motivation and its effect on patient outcomes. Maclean et al (2002) offer insights into the importance of patient motivation to recovery and how a perception that a patient is 'unmotivated' can affect the treatment they receive [27]. Karen et al (2004) looked at the more general field of exercising to promote healthy ageing and identified barriers of relevance for repetitive rehabilitation exercises [19]. These included pain from co-morbidities, negative views about exercise such as it being a 'waste of time' or 'unladylike', environmental barriers such as cost of exercise, a lack of specific advice from their GP and neighborhood safety. Recommendations to improve motivation for regular exercise included common themes such as forming habits, goal setting, monitoring progress, prompting, and incorporating instrumental music. Some studies show that an external focus is beneficial to motivation $[3,42]$. We explored clinicians' views on improving patient motivation, barriers to motivation and the role of technology in aiding recovery.

We looked at existing systems designed for motivation in various ways. We found numerous papers based on different definitions, theories, technologies and methods such as the Virtual Mall (VMall) project [38] that motivated stroke patients to engage in daily living activities such as shopping at a supermarket. A number of systems for stroke and motor rehabilitation have been developed for hospital settings, e.g. virtual environments [39]; mixed reality technology [37]; and tangible interfaces [45] and some are available commercially [12]. There are far fewer examples for the home setting where there are many technical and design challenges $[2,44]$. We considered which aspects could be transferred to the home.

We explored assistive systems such as a robotic arm used as a tool for stroke rehabilitation These use the manipulative ability of a robot and the incorporated sensor technologies to guide limb motion. They have been found to lead to large improvements of recovery of upper limb function including strength gains, reach extent, movement quality and patient motivation and $[9,24,29]$. The three main robotic devices used in randomised control trials are the ARM Guide ${ }^{\mathrm{TM}}$ (Assisted Rehabilitation and Measurement guide), Interactive Motion Technologies, Inc, Cambridge, MA), MIT-MANUS ${ }^{\mathrm{TM}}$ (Massachusetts Institute of Technology; and MIME $^{\mathrm{TM}}$ (Mirror-Image Motion Enabler) [22]. Even greater benefits might result if patients are intentionally moving their limb. In a review of the electrical stimulation literature [13] one of the main findings was that the effect of stimulation is enhanced when associated with the person's intention to move.

We looked for the clinical evidence base for using 'virtual' rehabilitation [7]. Holden and Dyar (2002) provide evidence of variable improvements of upper extremity function including strength for eight chronic post-stroke patients using virtual reality technology [17]. Movements of a virtual teacher are pre-recorded as movement templates and patients are asked to repetitively imitate upper limb rehabilitation. Significant aspects included the teacher-patient relationship, speed of motion, frequency of visual feedback, and degree of movement synchronization. Smart rehabilitation devices to monitor muscle activity hold promise [30]. Other work suggests that using real artifacts is better than using virtual ones [25, 46] $\mathrm{Ma}$ et al (1999) found participants learnt movements for eating with chopsticks better when learning with real food rather than simulated food [25].

We explored ways to monitor and measure movements. Monitoring is important because of the risk of abnormal movements leading to more physical disability. Patients might be tempted to use their 'good' hand and we could utilize restraints to prevent this[41]. Studies, such as the SMART project [49]. Zheng, Davies et al (2006) have made use of the Xsens $^{\mathrm{TM}}$ (Motion Technologies). MTX inertial sensors have good accuracy for home-based movement monitoring [48] This influenced prototype choices to show at our workshops.

Once monitored we must feed information back to various parties. Ambient feedback systems hold promise, such as the Virtual Aquarium and Mona Lisa Bookshelf [33]. Others highlight important ethical issues about feedback, such as ownership of the data resulting from monitoring systems [16].

We reviewed relevant human computer interaction literature. Haptic (touch) user interfaces hold promise for us as the majority of research to date has focused on using haptics with upper limb proprioception. Studies have investigated using haptic systems as part of the rehabilitation process. Amirabdollahian et al (2003) incorporated haptics into their Gentle/s system of upper limb rehabilitation along with some simple features to try and improve motivation [1]. Several studies have looked at using different types of haptic content to improve motivation. Most systems involve games $[5,6]$ or daily living tasks $[21,31]$. Other studies assess the benefits of collaboration to improve motivation $[18,23]$.

We considered potential barriers to the success of deployment. Colombo et al's robotic arm is not suitable for real homes as it is large, expensive and requires a clinician to set up [9]. Their simplistic on-screen tasks may lack appeal in the long term (their trial lasted only 3 weeks). Even if a system is demonstrated to promote therapeutic movements, if it is not acceptable to patients, therapists and budget holders, it will either not be deployed or will be used infrequently and so not be effective. Technology transfer problems have arisen with existing technological systems such as Functional Electrical Stimulation (FES) which currently reaches only a small fraction of potential patients. To minimise problems future developments (clinical and service provision research, technological development, and commercialisation) must meet all user needs and increase adherence with systems.

\section{PROTOTYPE DEVELOPMENT}

Ideas for prototypes were inspired and generated by close collaboration between those living with stroke, allied health professionals, designers and engineers. They were seeded early in our project when we carried out user studies and were developed and refined in the light of our exploration of related work, with particular emphasis on therapeutic, motivational and practical aspects. As promotion of therapeutic movements is the main system aim, we began with a clinically-inspired matrix categorization of different types of upper limb disability. This defined type of movement (activity classes) and ability level as shown in Figure 1. 


\begin{tabular}{|lc|c|c|c|}
\hline $\begin{array}{l}\text { Abilitylevel } \\
\boldsymbol{}\end{array}$ & $\begin{array}{c}\text { Activity } \\
\text { class } \rightarrow\end{array}$ & $\begin{array}{c}\text { Shoulder / } \\
\text { elbow }\end{array}$ & $\begin{array}{c}\text { Grasp / } \\
\text { release }\end{array}$ & Both \\
\hline Low & $\mathbf{x}$ & & \\
\hline Medium & $\mathbf{x}$ & $\mathbf{x}$ & $\mathbf{x}$ \\
\hline High & $\mathbf{x}$ & $\mathbf{x}$ & $\mathbf{x}$ \\
\hline
\end{tabular}

Figure 1. Clinical matrix (simplified)

We brought the matrix alive and inspired our design by developing a set of fictionalized case histories, (one for each matrix cell), inspired by the use of personas [11]. These anonymised histories of stroke patients were based on caseloads of clinical team members or from home visits earlier in our project. Personas reflected the diversity of people with stroke and illustrated their lives and requirements. We used collages of images to capture individual lifestyles, motivations and interests, combined with a brief clinical case history summary of functional problems and rehabilitation needs.

For each persona at least one design idea was developed to fit to their interests, motivations and functional rehabilitation requirements. Each design had a customized input device and potential monitoring system fitted to functional requirements, interactional content and feedback mechanisms fitted to personal interests and motivation. Input devices afforded therapeutically desirable upper limb movements. For example for one individual needing to regain elbow / shoulder control we designed a flat, touch-sensitive mat. Sliding a hand over the mat controlled an on-line chess game against a real opponent and necessitated repeated rehabilitative limb movements.

Having gathered design ideas, we selected one from each column of the functional matrix to develop into a low fidelity prototype to communicate our design ideas. We used a variety of resources to show how functional versions might operate, such as story boards, cartoon strips, video prototypes [4], acting out use (inspired by forum theatre 35] and cardboard models.

\section{STRUCTURE AND DESIGN OF WORKSHOPS}

Workshops took place in spring 2008 and gave us feedback on design ideas. Detailed information about the project aims and workshops structure was provided and informed consent was obtained.

\section{A. Structure of workshop for people with stroke}

One workshop was for people living with stroke and was attended by three couples, each with one partner who had a stroke and a carer who had not. Participants with stroke were recruited via their usual health or social care professional contact from a variety of organizations, including a private physiotherapy centre and stroke clubs.

We began with two parallel discussions led by members of the project. One discussion was for our participants who had a stroke and one was for their partners. Carrying out discussions in parallel allowed people to express their viewpoints without influence from partners and saved time, which was important in order to minimize chronic fatigue associated with stroke. Discussion was lively and participants were keen to share their experiences with us.
After the parallel discussions, couples toured a series of workstations where we demonstrated technology prototypes inspired by earlier research. Participants offered feedback and discussed what kinds of technologies they wanted at home. (In this paper, we do not focus on the detailed design of prototypes). Having seen demonstrations participants were asked to comment and audio recordings captured for later analysis. The concluding researcher-led discussion invited reflections on the workshop, and briefly discussed the future of the project and future opportunities for user involvement.

\section{B. Structure of workshops for professionals}

Our other two workshops were attended by about thirty stroke professionals from a variety of backgrounds including physiotherapists and occupational therapists. Professionals were identified from the register of the Association of Chartered Physiotherapists special Interest group in Neurology (ACPIN) They and their associates were invited by letter. We organized transport to ensure that people with impairments were not precluded from attending. Evening workshops were organized for professionals to fit local working practices. The two workshops for professionals shared a similar structure but took place in different parts of the UK. We made some small changes in the second workshop as we developed our process and better understood the needs of our participants.

As fatigue was less of a constraint we began with a talk by a member of the project to provide a general introduction and workshop aims. In the second of the workshops participants were split into small groups, each led by a coordinator who stimulated discussion about therapeutic practice. As in previous workshops, people toured technology prototype workstations, and ended with guided discussion and reflection. We asked therapists how prototypes might fit into professional practice.

\section{Data recording procedures}

Discussions throughout workshops were audio-recorded, to allow transcription. With each prototype was a set of post-it notes for participants to write down immediate thoughts and feelings. Field notes were taken at each workstation. This proved particularly useful with those living with stroke and a dedicated scribe worked with each couple. After the workshops recordings were uploaded and cleaned using Audacity to remove noise and adjust them to a common volume level. They were arranged in chronological order distributed across multiple tracks to allow transcribers to easily switch between them. Early recordings had poor sound quality that we were later able to improve by more careful use of physical space.

\section{DATA ANALYSIS}

Workshops gave us a significant amount of data and the analysis process took some time. Our methodology was primarily thematic. We identified common themes in our data and built descriptors for them. Our focus was to identify themes relating to both the social context of stroke and specific responses to technologies. Resulting themes are presented in this section and provide justification for our later discussion. We have chosen not to differentiate between stakeholders from different workshops. 


\section{A. Themes relating to participants' background information}

1) The disruptive affects of stroke

The elderly couples that attended our workshop told us how stroke disrupted their lifestyles. All reported significant changes in their relationships after stroke. One described how they had lost their "own little world". Several talked about how roles established over time that changed. One had to phone an in law to find out how to turn the oven on, having never before cooked a meal. All struggled with physical impairment and enforced change in environments. One couple had to move home, others made significant changes to homes, installing railings, alarms, or stair-lifts. The continuing need to care was an additional pressure, described as a " $24 / 7$ job", caring "from $6 \mathrm{am}$ to $10 \mathrm{pm}$ ". Time was needed to adjust and stress, anxiety and pressure were felt e.g. becoming "withdrawn". All had tried subsidized social care to help cope but, this was described as insufficient, inconsistent and low quality e.g. care staff arriving irregularly and failing to carry out tasks well, or at all.

2) The contribution of professionals to recovery

Professionals and those living with stroke contributed to discussion about therapy for recovery. Motivation for rehabilitation was considered of great importance and was encouraged in a number of ways. Therapists described performing continuous assessment of their patients to identify functional goals of importance to their patients and strategies to assist recovery. This included finding exercises of interest, helping identify the correct level of exercise, monitoring progress and explaining benefits of exercise to patients. Therapists also sub-divide large goals (such as learning how to walk again) into smaller, more quickly achievable goals (such as being able to stand, unassisted, for one minute). Observing and communicating progress to patients is very important. Therapists described patients' tendency to focus on functions they could not perform, rather than those regained and patients were felt to have poor recall of improvements they had made. This seemed to relate to the slow pace of stroke recovery that can be difficult for those living with stroke, day by day, to see.

\section{3) Limitations associated with therapy}

Therapy as a resource was significantly limited. Acute wards for those in early stages of stroke recovery had most resources with up to four sessions per patient per week, lasting twenty to forty minutes, focusing on recovery of lower limb mobility. Community therapists had fewer resources, providing roughly one session a week. After leaving community rehabilitation programs, therapy was rarely available at all and at this stage most were reliant on non-therapeutic care provided through local councils or their own resources.

Social support outside of therapy time seems really important in continuing recovery. People described the benefits of joining stroke groups or clubs "sitting round a table and ... talking about the things we were experiencing". Support from partners was important and several assisted with rehabilitation exercises and even policed the activity to ensure that it was carried out. Therapists described the importance of involving partners and training them to help since even when patients were involved in treatment programs the vast majority of their time was spent at home.

\section{B. Participant responses to technology prototypes}

Reactions to our technology prototypes were generally positive. They facilitated a lot of discussion in all workshops.

\section{1) Content for technologies}

There was substantial agreement between therapists and couples that content is really important and must be tuned to the interests and needs of individual patients with a range of choice to avoid boredom. They envisaged a bank of hardware and software with a library of content. Therapists suggested deployment would create a need for a new professional role - a system manager, to source, provide and maintain this bank and provide advice for patients and therapists. Constraints on resources dictate that systems should be able to run on relatively low-powered computing technology, possibly including computers refurbished by charities. A number of suggestions were made on content including access to resources to help build family trees and accessing photos. One focus was on traditional games, such as chess, draughts and backgammon and playing remotely with others over network connections was a popular idea. Stroke and other age-related disabilities had restricted social lives and so providing added social interaction was important. Therapists suggested homeshopping as a useful computer based practical activity but those living with stroke disagreed, describing shopping as an important motivation to get out of the house.

\section{2) Technology design issues}

Designing technology to suit those living with stroke and their therapists is important and challenging for a variety of reasons. Co-morbidities often associated with age are a constraint. One participant found reading information from a screen difficult due to visual problems. Several suggested it might be easy to ignore a computer system and not engage with exercises. Several described times when system use would be inappropriate, e.g. due to levels of fatigue (which are commonly associated with stroke) in the evening. One described inability to extend her arm as much in the evening and another described the evening as being "a long descent".

Therapists pointed out that systems should strike the right balance or they might prove de-motivating. They should provide significant challenge but be achievable. Physical abilities vary from day to day and even during the course of just one session. Systems might need to automatically adapt to these varying levels of ability. Many patients had cognitive impairments due to stroke or normal aging. Therapists advised avoiding interfaces involving too much abstract thought. Using a mouse to control an on-screen computer game was considered abstract. Functionality should be as close as possible to real games, e.g. a real chess board as an input device, rather than an on-screen chess board. In coming decades a generation of people more adept with computer technology will reach old age and may have sufficient computer skills for current technologies. But with an exponential rate of technology change along with a tendency for older people to have less fluid and more crystallized knowledge, it may be that older users will always find it challenging to use new technologies, whatever the current state of the art is. 


\section{3) Therapeutic issues in technology deployments}

Therapists perceive risks to patients from performance of 'abnormal' movements when unsupervised in the home environment. Compensatory movements can be damaging or can slow recovery (e.g. misalignment of the trunk by twisting or rotating in order to increase reaching distance). If content was so interesting that people stopped thinking about how they were carrying out movements, they might use more compensatory movements or forget to use the affected limb and use their 'good arm instead. Several solutions were suggested. Careful system design and deployment could enforce 'good' movements or restraints could prevent the use of the 'good' hand. Automatic sensing could detect compensations and the system could respond with instructions to correct the movement or could shut down to prevent repeated undesirable movements. Patients could request help. Patients are always at risk of performing abnormal movements at home as part of their daily living activities. A system with feedback to both therapist and patient about movement patterns could highlight problems and offer a mechanism for correction. Compensatory movements are most likely when patients are tired so a system to monitor fatigue and encourage periods of engagement and rest might reduce risks. Deployment in supervised environments such as stroke or social clubs with peers or staff to provide feedback and system use might mitigate risk.

\section{4) Other issues around the use of the system}

Despite broadly positive reactions to our ideas Partners of those with stroke worried that a system might add to the burden of their already pressured lives. One participant was worried he might not be able to fix a system if it broke. Another hoped a robust and interesting system, used independently, might give him more time to himself. Some were worried patients might try and "cheat" when using the system (e.g. using the 'good" arm rather than the impaired limb). Patients offered solutions such as "sitting on my good hand" (suggested to her by a therapist) or restraints such as wearing a heavy glove. Partners suggested they could play an enforcing role. Sensors built into a system could provide feedback to all parties about hand use.

\section{IV.DISCUSSION}

Through analysis of workshop contributions we have increased our understanding of the challenges of designing effective computer systems to aid in rehabilitation of those who have experienced stroke. In this discussion we consider the key themes and requirements for home-based rehabilitation technologies. This requirements analysis is the primary contribution of this paper. We start with a discussion of the wider issues of designing for the social context that exists around stroke and then discuss specific features identified through our analysis.

\section{A. Designing for wider social context of strokes}

Discussions in our workshops illustrated issues around the availability of resources for stroke treatment and the impact of this. Therapist contact is limited and therapists felt their impact outside of therapy sessions was even more limited. This was increasingly true for patients several years post stroke when therapy contact was usually not available at all. The provision of some therapeutic support via a computer system in the home has potential for significant benefit. Such a system should never replace a therapist but may extend their reach and influence beyond the therapy session or enable therapeutic influence for those who have no ongoing interaction with health professionals. A home based therapeutic system would be a useful addition to the existing range of tools and resources.

In our workshops we identified a number of challenges about design and deployment for those who have experienced stroke. In terms of requirements for systems it is important to note the significant levels of pressure due to stroke that all participants reported. Partners in particularly wanted to avoid creating any additional pressures in their lives. Issues around caregiver strain were also indicated in CareNet Display [10] Current social care structures were perceived as inadequate and unlikely to contribute support to deployment of a system. It is important to design a system that can stand alone without substantial outside support. The system should be very robust and require little installation or maintenance effort. Any future larger scale deployment would need to consider this issue. New social or therapy support structures might be required such as trained organizers to administer deployment and support.

In terms of the design process itself, it is important not to underestimate the difficulty of designing systems with participants who may have extensive mental and physical impairments, and may not be at all familiar with the capabilities of computers. We have identified five important stakeholder groups: patients, partners or relatives, paid carers, therapists and health and social care budget holders. All have very different requirements and ideas about what the system is for and how it should perform. Satisfying all these groups is an interesting challenge with which we plan to engage. Financial constraints due to limited personal and health and social care budgets mean that a successful system design will have to make use of relatively cheap, commodity technology. Designing a system that is genuinely therapeutic that both affords and monitors 'good' movements is an interesting challenge. One clear learning point from the workshops was that any system deployed must be individualized to be interesting, enjoyable and motivating to those who are using it.

\section{B. Specific technology features identified at workshops}

\section{1) Personalising system components}

Input device requirements will vary depending upon the individual physical functional ability of the person and their technological ability. Functional ability may alter over time as their condition improves or deteriorates. Some might have a flaccid or spastic limb requiring support to move, some might have difficulties with grasping objects and need something of a particular size, weight or texture to hold. Possible range of movement and reach will vary. In traditional physiotherapy sessions goal setting activities are regularly carried out and targets reviewed and re-set. On the technological side, some might enjoy using computer technologies such as desk top with screen and mouse while others would find this intimidating and benefit more from 'hidden' technologies embedded in their everyday environment. If input devices intended to provide for control of content are too easy or difficult to use they could be de-motivational. This is a design challenge as abilities may change over even just the course of one session. 
Requirements for application content will vary from person to person, depending on interests and experiences e.g. traditional games had appeal to all but there were a wide variety of preferences within this. They may change from day to day depending on mood or changing social contexts. Existing therapeutic practices and resources might provide support for the process of selection and personalization of system components. Therapists told us how they establish the work and leisure interests of their patients and design exercise schedules to be interesting and engaging to individuals. We hope to capture this process within our system.

As our project resources are limited we plan to build and personalize for a small number of individuals in the short term. In the long term we hope to assemble a library of input devices application content, monitoring devices and feedback mechanisms from which patients and therapists can select. We hope to adapt and/or link to existing libraries of content, some of which are freely available as shareware. We are currently developing a general purpose software framework to facilitate integration of this kind of content into a rehabilitation system.

Managing and structuring large libraries of system components is an interesting challenge. Within our system we might ultimately offer a range of input mechanisms, application content and feedback mechanisms. We envisage gradually developing a bank of resources and indexing each item for a number of variables such as patients' interests, cognitive abilities, performance of available hardware, availability of space in the home and availability of additional resources such as networking support. In practice therapists felt such a resource would require the creation of a new professional role to manage it, as therapists would not have sufficient time or experience to take on the responsibility. The skills, training and experience required for such a role is an issue for future research, alongside practical system implementation and use.

\section{2) Initiating and concluding interactions with users}

Our aim in seeking to design personalized systems with interesting, engaging and motivating content is to encourage exercise through the use of specialized input devices. Some patients find the idea of technology worrying. They may need a system which does not appear 'technological' or careful management to cope with adoption of a system. The system may need features to encourage them to initiate interaction and exercise and we have various design options to consider.

The least intrusive and most autonomous option is to deploy a system that requires the patient to decide to begin interaction and to move to wherever in their home the system is deployed. We could simplify the process by providing a simple mechanism such as a large button for patients to push to start the system. Patients could interact with content until either the system determines that enough exercise has been done or the patient decides to stop.

For those who are not self-motivated systems might have a mechanism to alert patients that it is time for exercise. We could involve artifacts that already exist in their environment (such as making a desk lamp flash) or add new technology features to the home. Our workshop data indicated that certain times of the day were not conducive to system interaction due to the demands of daily living or due to fatigue. An interesting approach would be to develop a system that could learn the best times to initiate interaction.

Finishing cycles of engagement is important to motivation but might be prevented by fatigue, commonly encountered by those with stroke. To counter this we might allow patients to pause their activity and take a break. They may choose to terminate activity at any point or require feedback from the system at that point. The system could remind them where they had got to and suggest restart points.

3) Sensing and processing inputand quality of movement

Our workshop analysis shows that we need to support two different kinds of input to our system - input from a device to promote limb movement (which is used to control application content) and input from sensors indicating quality of movement. Both are required to ensure therapeutic value of the patient experience and patient motivation. A particular challenge is how to design system responses to 'poor' quality movements. Devices such as accelerometers and gyroscopes provide position/angle information for applications like navigation, position control of vehicles/aircrafts, and monitoring of daily-life activities [20]. These have been popular for home-based monitoring, as they do not suffer from the 'line-of-sight' problem which is still hard to effectively deal with in a home based environment. A major problem is how sensor readings can be erroneous (suffer from a fluctuating offset) and not correctly determined due to a temperature change, mechanical wear, or measurement noise which can lead to an integration drift. More recent developments in the computer games technology and virtual reality industry led to the release of a new generation of inertial sensors, composed of both accelerometers and gyroscopes. These sensors have been used to provide joint angle information for static and dynamic full-body activities in an easy-to-use and cost efficient way. Despite the inclusion of magnetometers into the sensors and devising drift correction techniques based on magnetic compass measurements the sensors still suffer from the common drift problem as compass signals are subject to errors induced by ferrous materials. Different approaches to handle these errors have been investigated e.g. vision-based corrections [47] and error models [34]. No sensor system can be perfect and careful design is needed so that false-positives (i.e. identifying good quality movements as poor quality) could cause significant problems.

\section{4) Providing feedback to users}

Theories of motor learning stress the positive correlation between feedback information (both type and frequency) and practice intensity with improved motor learning and skill acquisition [28]. Therapists indicated that providing the right feedback to patients is a key mechanism of practice. Feedback can be provided in many ways. Therapists provide feedback on performance of individual movements and overall progress made. Feedback is a topic of therapeutic research as to best models of feedback evidence of their success in use [8]. A consensus exists that positive feedback with a partial reinforcement schedule is motivational. There is evidence that self efficacy is important. Patients have poor awareness of their own progress and that performance of movements is improved by feedback that allows them to identify their own errors. 
In practice in home settings, away from clinical input feedback may be less than optimal. The lack of feedback may be one reason that, in general, motivation to perform exercises does not seem to be as high outside of therapy settings. Patients may receive feedback from partners, relatives or carers but such individuals are not experts in therapy and may offer either insufficient or inaccurate feedback. A system that could generate an appropriate positive partial reinforcement schedule, increase self efficacy and provide personalized interesting content should stimulate exercise practice and motivation.

The choice of feedback regimes would be constrained by cost and complexity of integration and the needs and desires of the individual stakeholders. Immediate feedback to the patient while exercises are being performed could provide advice about quality of movement. The generation of top quality feedback might be reliant upon patients wearing extensive sensors which may not be practical in a domestic setting. A simpler solution could depend on a more limited range of sensors deployed to detect the most risky movements. Feedback might consist of the system shutting down or offering advice such as playing a video to demonstrate good movements or offering peer comparison. Feedback displays could show measures of performance over time - perhaps the last week or the last month to support motivation. Graphs and statistics or ambient displays could be an option.

A critical moral and ethical issue relates to ownership of data and access to feedback. Budget holders might want to monitor use in order to ration deployment depending on progress. Carers or therapists could access feedback data to monitor progress and this might be useful for planning or supporting programmes of exercises. Patients might find that constantly having someone remotely "watching over your shoulder" is undesirable. Patients attending clinics are at liberty to disclose or not as to how much they have really practiced at home. Our system might need a design for plausible deniability in order to be socially acceptable. Further research is needed to work out the best designs for feedback systems.

\section{FRAMEWORK}

We will personalize systems and offer a variety of input, content and feedback mechanisms. The design of components will follow guidelines derived from motivational theories and evidence from physiotherapy practice to inspire motivation to use them. We will use innovative technologies and embed them into daily lives, routines and wider social networks so they are readily available, easy to use and fit to requirements and motivations of stakeholders.

Informed by our arguments Figure 2 illustrates the potential design framework for a socio-technical system to be installed into individual homes. This figure does not include components that relate to the content management database. We have not yet defined or determined the precise nature of the operation of these and consider this an interesting question for future research. We present the diagram to stimulate discussion about the nature of these kinds of systems.

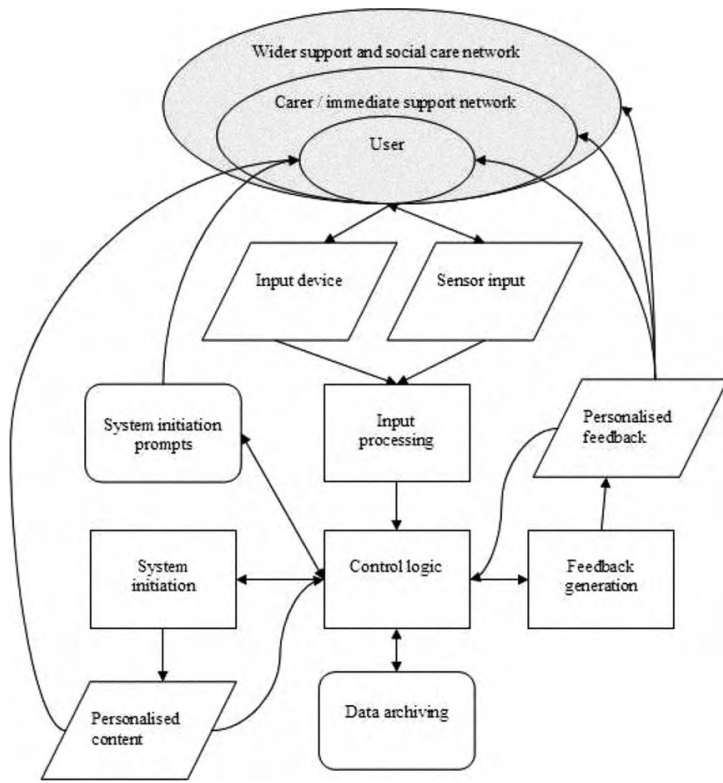

Figure 2 - workshop informed socio-technic design framework

\section{CONCLUSION}

Analysis of data from our three workshops has provided some interesting insights into the issues and challenges involved in developing technologies for use in the homes of those who live with stroke. As part of our participatory design philosophy and following individual user studies, it has allowed us to share low fidelity prototypes and sketch out interactions between a set of abstract components that may make up an eventual system. It has raised a significant number of questions for future research including work to determine the operation of these components and to validate their choice.

\section{ACKNOWLEDGEMENTS}

This project is funded by EPSRC grant EP/F00382X.

\section{REFERENCES}

[1] Amirabdollahian, F., Gradwell, E., Loureiro, R., Collin, C., and Harwin, W. (2003). Effects of the Gentle/s Robot Mediated Therapy on the Outcome of Upper Limb Rehabilitation Post-Stroke: Analysis of the Battle Hospital Data. Proc 8th IntConf RehabRobotics (ICORR 2003), HWRS-ERC Human-friendly Welfare Robot System Engineering Research Center, KAIST, Korea, pp. 55-58, April 23-25

[2] Attygalle, S., Duff, M., Rikakis, T. and He J. (2008) Low-cost, at-home assessment system with Wii Remote based motion capture, Virtual Rehabilitation 2008, Vancouver, Canada

[3] Bach- y-Rita P. et.al. Computer-Assisted Motivating Rehabilitation (CAMR) for Institutional, Home, and Educational Late Stroke Programs. Top Stroke Rehabil 2002;8(4):1?10

[4] Bardram, J., Bossen C., Lykke-Olesen, A., Madsen, K.H. \& Nielsen, R. Virtual Video Prototyping of Pervasive Healthcare Systems. The proceedings of DIS 2002, London: 2002 ( 167-177)

[5] Broeren J. et.al.: Virtual Rehabilitation in an Activity Centre for Community-Dwelling Persons with Stroke - The Possibilities of 3Dimensional Computer Games. Cerebrovasc Dis 2008;26:289?296 DOI: $10.1159 / 000149576$ 
[6] Broeren, J., Georgsson, M., Rydmark, M., \& Stibrant Sunnerhagen, K., Virtual reality in stroke rehabilitation with the assistance of haptics and telemedicine, in proc. $4^{\text {th }}$ Int Conf on Disability, VR and Associated Technologies (ICDVRAT 2002), Veszprm, Hungary, 2002

[7] Burdea G. C.. Virtual Rehabilitation? Benefits and Challenges. Methods Inf Med 2003; 42: 519?23

[8] Cirstea CM, Ptito A, Levin MF (2006) Feedback and Cognition in Arm Motor Skill Reacquisition After Stroke. Stroke 37 1237-1242

[9] Colombo R. et.al.: Design strategies to improve patient motivation during robot-aided Rehabilitation. Journal of NeuroEngineering and Rehabilitation 2007, 4:3 doi:10.1186/1743-0003-4-3

[10] Consolvo S, Roessler P, Shelton Be (2007) The CareNet Display: Lessons Learned from an In Home Evaluation of an Ambivalent Display. Inter Research Seattle. www.peterroessler.com/ubicompcarenet-final.pdf (accessed 27.02.09)

[11] Cooper, A. The inmates are running the asylum: why high-tech products drive us crazy and how to restore sanity. Published by SAMS, 1999. GestureTek Health (2009) http://www.gesturetekhealth.com (accessed 24 February 2009)

[12] De Kroon, J. R, IJzerman, M. J., Chae, J., Lankhorst, G. J., and Zilvold, G. (2005) Relation between stimulation characteristics and clinical outcome in studies using electrical stimulation to improve motor control of the upper extremity in stroke., J Rehab Medicine, 37 (2), pp. 65-74

[13] Dept of Health UK 2006 Our health, our care, our say: a new direction for community services.

[14] European Commission, 2005.Confronting demographic change: a new solidarity between the generations. Published by the http://eurlex.europa.eu/LexUriServ/site/en/com/2005/com2005 0094en0 1.pdf accessed (27.2.09)

[15] Green, W. 2008 The appropriateness of secrets and lies for socially intelligent tele-healthcare, CHI 2008

[16] Holden, M. and T. Dyar (2002). "Virtual environment training: a new tool for rehabilitation." Neurorehabil. Neural Repair 26: 62-71

[17] Johnson, M.J., Loureiro, R.C.V., and Harwin, W.S. (2008) Collaborative Tele-rehabilitation and Robot-Mediated Therapy for Stroke Rehabilitation at Home or Clinic. In Special issue Journal of Intelligent Service Robotics, Springer-Verlag,

[18] Karen A. Schutzer et.al: Barriers and motivations to exercise in older adults. Preventive Medicine $39 \quad$ (2004) 1056 ? 1061. doi:10.1016/j.ypmed.2004.04.003

[19] Kavanagh, J. J. and H. B. Menz (2008). "Accelerometry: a technique for quantifying movement patterns during walking (review)." Gait \& Posture 28: 1-15

[20] Kayyali, R., Shirmohammadi, S., El Saddik, A., \& Lemaire, E., DailyLife Exercises for Haptic Motor Rehabilitation, in proc. Haptic, Audio and Visual Environments and Games, 2007. HAVE 2007. IEEE International Workshop on, 12-14 Oct. 2007, pp. 118-123, 2007

[21] Kwakkel, G., B. J. Kollen, et al. (2008). "Effects of robot-assisted therapy on upper limb recovery after stroke: a systematic review." Neurorehabil. Neural Repair 22(2): 111-121

[22] Loureiro, R., Amirabdollahian, F., Coote, S., Stokes, E., and Harwin, W. (2001). Using Haptics Technology to Deliver Motivational Therapies in Stroke Patients: Concepts and Initial Pilot Studies. In Proceedings of the 1st European Conference on Haptics (EuroHaptics 2001), Educational Technology Research Paper Series, University of Birmingham, Birmingham, UK, pp. 1-6, ISSN 1463-9394.

[23] Lum, P. S., C. G. Burgar, et al. (2002). Robot-assisted movement training compared with conventional therapy techniques for the rehabilitation of upper-limb motor function after stroke. Arch Phys Med Rehabil 83: 952-959.

[24] Ma HI, Trombly CA, Robinson-Podol- ski C (1999) The effect of context on skill acquisition $a$ nd transfer.Am J Occ. Ther 53: 138-144

[25] Mackay J and Mensah G. (2004) Atlas of heart disease and stroke, World Health Organisation Publication: 51-55

[26] Maclean et.al:: The Concept of Patient Motivation - A Qualitative Analysis of Stroke Professionals? Attitudes. Stroke 2002;33;444-448. DOI: $10.1161 / \mathrm{hs} 0202.102367$
[27] Magill RA. Motor Learning: Concepts and Applications. 6th ed McGraw-Hill International Editions; 2001

[28] Masiero, S., A. Celia, et al. (2007). "Robotic-assisted rehabilitation of the upper limb after acute stroke." Arch Phys Med Rehabil 88: 142-149.

[29] Mavroidis, C., J. Nikitczuk, et al. (2005). "Smart portable rehabilitation devices." J. NeuroEng. Rehab. 2(18)

[30] McLaughlin, M., Rizzo, A., Jung, Y., Peng, W., Yeh, S., Zhu, W., and the USC/UT Consortium for Interdisciplinary Research (2005), Hapticsenhanced virtual environments for stroke rehabilitation. Proc. IPSI 2005, Cambridge, MA.

[31] McLean N., Pound P., Wolfe C. and Rudd A. (2002) ' The concept of patient motivation; a qualitative analysis of stroke professionals;', Stroke 33 444-448

[32] Millan M, Davalos A. (2006) The need for new therapies for acute ischemic stroke, Cerebrovasc Dis 22(suppl 1):3-9

[33] Nakajima, T., Lehdonvirta, V., Tokunaga, E., and Kimura, H. 2008 Reflecting human behavior to motivate desirable lifestyle. In Proceedings of the 7th ACM Conference on Designing interactive Systems (Cape Town, South Africa, February 25 - 27, 2008). DIS '08. ACM, New York, NY, 405-414.

[34] Nebot, E. and H. Durrant Whyte (1999). "Initial calibration and alignment of low-cost inertial navigation units for land vehicle applications." Journal of Robotic Systems 16(2): 81-92.

[35] Newell, A.F., Carmichael, A. Morgan, M., Dickinson, A: The use of theatre in requirements gathering and usability studies. Interacting with Computers, Vol. 18, 2006. pp. 996- 1011

[36] Pomeroy V.M; Tallis R.C. (2002) Restoring movement and functional ability poststroke: now and the future. Physiotherapy, 88, 3-17.

[37] Pridmore, T., Cobb, S., Hilton, D., Green, J. and Eastage, R. (2007) Mixed reality stroke rehabilitation: interfaces across the real/virtua divide, Intl. Journal of Disability\& Human Development, 6,; 1, pp. 3-10

[38] Rand, D., M. Katz, et al. (2004). The virtual mall: development of a functional virtual environment for stroke rehabilitation. The 55th Annual Conference of the Israel Association of Physical \& Rehab Med.

[39] Sveistrup, H. (2004). Motor rehabilitation using virtual reality, Journal of NeuroEngineering \& Rehabilitation, Vol. 1, No. 10, pp. 1-8

[40] Tuke A (2008) Constraint-induced movement therapy: a narrative review. Physiotherapy 94 105-114 www.dh.gov.uk/en/Publicationsandstatistics/Publications/PublicationsPo licyAndGuidance/DH 4127602 (accessed 27.2.09)

[41] Van Vliet P, Wulf G. Extrinsic Feedback for Motor Learning after Stroke: What is the evidence? Disability and Rehabilitation 2006;28:831-840

[42] WHO Neurological Disorders: Public Health Challenges (2006) www.who.int/mental health/neurology/neurodiso/en/print.html (accessed 27.2.09)

[43] Willmann, R., Lanfermann, G., Saini, P., Timmermans, A., Vrugt, J. and Winter, S. (2007) Home stroke rehabilitation for the upper limbs, Conf. Proc. IEEE Eng. Med. Biol. Soc. 2007, pp. 4015-4018

[44] Wilson, P., Duckworth, J., Mumford, N., Eldridge, R., Guglielmetti, M., Thomas, P., Shum, D. and Rudolph, H. (2007) A virtual tabletop workspace for the assessment of upper limb function in Traumatic Brain Injury (TBI), Proc. of Virtual Rehabilitation 07, Venice, Italy, pp. 14-19

[45] Wu C., Trombly C., Lin K., Tickle-Degnen L. A kinematic study of contextual effects on reaching performance in persons with and without stroke: influences of object availability. Arch Phys Med Rehabil 2000 81: 95-101

[46] You, S., U. Neumann, et al. (1999). Hybrid inertial and vision tracking for augmented reality registration. Proc. of IEEE VR'99, pp.260-267

[47] Zheng, H., R. Davies, et al. (2006). The SMART Project: An ICT decision platform for home-based stroke rehabilitation system Proceedings of the International Conference on Smart homes and Telematics (ICOST).

[48] Zhou, H. and H. Hu (2008). "Human motion tracking for rehabilitation a survey." Biomedical Signal Processing and Control 3: 1-18. 